Supporting Information to

\title{
Bionic Thermoelectric Response with Nanochannels
}

\author{
Kexin Chen, Lina Yao and Bin Su* \\ Institute of Analytical Chemistry, Department of Chemistry, Zhejiang University, \\ Hangzhou 310058, China \\ * Corresponding Author: Dr. Bin Su \\ Email: subin@zju.edu.cn
}

\section{Table of Contents}

S1. Theoretical Derivation of Thermoelectric Response

S2. Preparation of SIM/PET Hybrid Nanochannels

S3. I-V Response of Nanochannels

S4. Thermoelectric Response of PET Conical Nanochannels

S5. Thermoelectric Response at a Low pH

S6. Redox Potential Difference of Two $\mathrm{AgCl} / \mathrm{Ag}$ Electrodes

S7. Numerical Simulations 


\section{S1. Theoretical Derivation of Thermoelectric Response}

The model shown in Figure $1 \mathrm{a}$ is considered to derive the theoretical thermoelectric response. Two solutions, designated as $\alpha$ and $\beta$, respectively, contain 1:1 electrolyte $(\mathrm{KCl}$ or $\mathrm{NaCl})$ at a concentration of $c^{\alpha}$ and $c^{\beta}$. In this work, $\mathrm{KCl}$ or $\mathrm{NaCl}$ was used as the electrolyte. So the subscripts, + and -, refer to $\mathrm{K}^{+} / \mathrm{Na}^{+}$ and $\mathrm{Cl}^{-}$(the same in the following other symbols), respectively. Accordingly, the temperature of two solutions is $T^{\alpha}$ and $T^{\beta}$. Considering a quasi-steady state of ion transport through nanochannels from one solution to another when the temperature gradient is present, we have the following equation,

$$
t_{+} \bar{\mu}_{+}^{\beta}+t_{-} \bar{\mu}_{-}^{\alpha}=t_{+} \bar{\mu}_{+}^{\alpha}+t_{-} \bar{\mu}_{-}^{\beta}
$$

where $t_{i}$ is the transference number of ions. $\bar{\mu}_{i}^{j}(j=\alpha, \beta)$ is the electrochemical potential of ions and given by,

$$
\bar{\mu}_{i}^{j}=\mu_{i}^{\ominus, j}+R T^{j} \ln a_{i}^{j}+z_{i} F \phi^{j}(j=\alpha, \beta)
$$

where $\mu_{i}^{\oplus, j}, a_{i}^{j}$ and $z_{i}$ are the standard chemical potential, the activity and the charge valence of ions. $R$ and $F$ are the gas constant and the Faraday constant, respecitvely. $\phi^{j}$ is the inner potential of two solutions.

In a dilute aqueous solution (10 $\mathrm{mM}$ in the present work), it can be supposed that the ion concentration is equal to its activity, namely $c_{i}^{j}=a_{i}^{j}$, given the activity coefficients of all ions is close to $1\left(a_{\mathrm{K}^{+}}=, a_{\mathrm{Na}^{+}}=\right.$and $a_{\mathrm{Cl}^{-}}=$at 298.15 K). Note that the variation of activity coefficients with temperature is also neglected here, due to a small temperature change (1o K, maximum) in this work. Then eq. $\mathbf{S} 2$ can be rewritten as,

$$
\bar{\mu}_{i}^{j}=\mu_{i}^{\Theta, j}+R T^{j} \ln c_{i}^{j}+z_{i} F \phi^{j}(j=\alpha, \beta)
$$

Considering the electroneutrality condition in two solutions, we have,

$$
c_{+}^{\alpha}=c_{-}^{\alpha}=c^{\alpha}, \quad c_{+}^{\beta}=c_{-}^{\beta}=c^{\beta}
$$


In this case, from above four equations we can derive the following one,

$$
\left(t_{+}-t_{-}\right) R T^{\beta} \ln c^{\beta}+\left(t_{-}-t_{+}\right) R T^{\alpha} \ln c^{\alpha}=F\left(\phi^{\alpha}-\phi^{\beta}\right)
$$

Then we can define the trans-nanochannel diffusion potential $\left(\phi_{\text {diff }}\right)$ as,

$$
\phi_{\text {diff }}=\left(\phi^{\alpha}-\phi^{\beta}\right)=\left(2 t_{+}-1\right)\left(\frac{R T^{\beta}}{F} \ln c^{\beta}-\frac{R T^{\alpha}}{F} \ln c^{\alpha}\right)
$$

According to eq. S6, $\phi_{\text {diff }}$ is a function of both temperature and salt concentration in two solutions.

Figure $\mathbf{l b}$ shows the equivalent circuit of experimental system, in which the the open-circuit potential $\left(V_{\text {oc }}\right)$ is measured with two silver/silver chloride $(\mathrm{Ag} / \mathrm{AgCl})$ electrodes immersed in two solutions. It is the sum of thermoresponsive trans-nanochannel potential ( $\phi_{\text {diff }}$, the direction from $\alpha$ to $\beta$ is defined to be positive), the difference of redox potentials of two $\mathrm{AgCl} / \mathrm{Ag}$ electrodes $\left(E_{\text {redox }}\right)$ and the voltage drops across nanochannel membrane $\left(i R_{\text {channel }}\right)$ and in solution $\left(i R_{\mathrm{s}}\right)$,

$$
V_{\mathrm{oc}}=-\left(\phi_{\text {diff }}+E_{\text {redox }}+i R_{\text {channel }}+i R_{\mathrm{s}}\right)
$$

where $i$ is the ionic current, $R_{\text {channel }}$ the internal resistance of nanochannels and $R_{\mathrm{s}}$ the solution resistance. Considering the ionic current measured is in the $\mathrm{nA}$ range, we can reasonably neglect the contribution of $i R$ drop. $E_{\text {redox }}$ arises from the dependence of $\mathrm{AgCl} / \mathrm{Ag}$ electrode potential on the concentration of $\mathrm{Cl}^{-}$and temperature,

$$
E_{\text {redox }}=E_{\text {redox }}^{\alpha}-E_{\text {redox }}^{\beta}=\frac{R T^{\beta}}{F} \ln c^{\beta}-\frac{R T^{\alpha}}{F} \ln c^{\alpha}
$$

Combining eqs. S6, $\mathbf{S} 7$ and $\mathbf{S 8}$, we obtain the following equation,

$$
V_{\mathrm{oc}}=-\left(\phi_{\text {diff }}+E_{\text {redox }}\right)=-2 t_{+}\left(\frac{R T^{\beta}}{F} \ln c^{\beta}-\frac{R T^{\alpha}}{F} \ln c^{\alpha}\right)
$$


In this work, we studied the thermoelectric response for two cases, in the absence and presence of concentration gradient across nanochannels. In the first case, there is no concentration gradient across nanochannels (namely $c^{\alpha}=c^{\beta}=c$ ). Both $\phi_{\text {diff }}$ and $V_{\text {oc }}$ are equal to zero at the initial state $\left(T^{\beta}=T^{\alpha}\right)$. Upon changing the temperature of one solution (the temperature of another solution remained unchanged), they will vary and the magnitudes can be derived from eqs. S6 and S9, respectively,

$$
\begin{aligned}
& \Delta \phi_{\text {diff }}(T)=\left(2 t_{+}-1\right) \frac{R}{F} \Delta T \ln c \\
& \Delta V_{\text {oc }}(T)=-2 t_{+} \frac{R}{F} \Delta T \ln c
\end{aligned}
$$

where $\Delta T\left(\Delta T=T^{\beta}-T^{\alpha}\right)$ is the magnitude of temperature change, namely the immediate temperature difference between two solutions separated by nanochannels.

In the second case (namely $c^{\alpha} \neq c^{\beta}$ ), there exist nonzero $\phi_{\text {diff }}$ and $V_{\text {oc }}$ at the initial state. If assuming the initial temperature of two solutions is $T_{\mathrm{o}}$, they are associated with the concentration gradient and expressed as,

$$
\begin{aligned}
& \phi_{\text {diff }}(0)=\left(2 t_{+}-1\right)\left(\frac{R T^{0}}{F} \ln c^{\beta}-\frac{R T^{0}}{F} \ln c^{\alpha}\right) \\
& V_{\text {oc }}(0)=-2 t_{+}\left(\frac{R T^{0}}{F} \ln c^{\beta}-\frac{R T^{0}}{F} \ln c^{\alpha}\right)
\end{aligned}
$$

In the similar way, when the temperature of one solution was changed (the temperature of another solution remained unchanged), both $\phi_{\text {diff }}$ and $V_{\text {oc }}$ will change. Their net variations relative to the initial state are dependent on both temperature and concentration gradients and can be expressed as, 


$$
\begin{aligned}
\Delta \phi_{\text {diff }}(c, T) & =\phi_{\text {diff }}(c, T)-\phi_{\text {diff }}(0) \\
& =\left(2 t_{+}-1\right)\left(\frac{R T^{\beta}}{F} \ln c^{\beta}-\frac{R T^{\alpha}}{F} \ln c^{\alpha}-\frac{R T^{0}}{F} \ln c^{\beta}+\frac{R T^{0}}{F} \ln c^{\alpha}\right) \\
& =\left(2 t_{+}-1\right) \frac{R}{F} \Delta T \ln c^{\mathrm{T}} \\
\Delta V_{\mathrm{oc}}(c, T) & =V_{\mathrm{oc}}(c, T)-V_{\mathrm{oc}}(0) \\
& =-2 t_{+}\left(\frac{R T^{\beta}}{F} \ln c^{\beta}-\frac{R T^{\alpha}}{F} \ln c^{\alpha}-\frac{R T^{0}}{F} \ln c^{\beta}+\frac{R T^{0}}{F} \ln c^{\alpha}\right) \\
& =-2 t_{+} \frac{R}{F} \Delta T \ln c^{\mathrm{T}}
\end{aligned}
$$

where $c^{T}$ is the electrolyte concentration in the solution where the temperature changes. Note that $\Delta T\left(\Delta T=T^{\beta}-T^{\alpha}\right)$ is defined as above and denotes the magnitude of temperature change. For instance, if the temperature of $\alpha$ is changed while that of $\beta$ is kept constant (namely $c^{\mathrm{T}}=c^{\alpha} ; T^{\beta}=T^{\mathrm{o}}, \Delta T=T^{\beta}-T^{\alpha}$ $\left.=T^{\mathrm{o}}-T^{\alpha}\right)$, we have,

$$
\begin{aligned}
& \Delta \phi_{\text {diff }}(c, T)=\left(2 t_{+}-1\right)\left(T^{0}-T^{\alpha}\right) \frac{R}{F} \ln c^{\alpha}=\left(2 t_{+}-1\right) \frac{R}{F} \Delta T \ln c^{\mathrm{T}} \\
& \Delta V_{\text {oc }}(c, T)=-2 t_{+}\left(T^{0}-T^{\alpha}\right) \frac{R}{F} \ln c^{\alpha}=-2 t_{+} \frac{R}{F} \Delta T \ln c^{\mathrm{T}}
\end{aligned}
$$

In the opposite (namely $c^{T}=c^{\beta} ; T^{\alpha}=T^{\mathrm{o}}, \Delta T=T^{\beta}-T^{\alpha}=T^{\beta}-T^{\mathrm{o}}$ ), we have,

$$
\begin{aligned}
& \Delta \phi_{\text {diff }}(c, T)=\left(2 t_{+}-1\right)\left(T^{\beta}-T^{0}\right) \frac{R}{F} \ln c^{\beta}=\left(2 t_{+}-1\right) \frac{R}{F} \Delta T \ln c^{\mathrm{T}} \\
& \Delta V_{\text {oc }}(c, T)=-2 t_{+}\left(T^{\beta}-T^{0}\right) \frac{R}{F} \ln c^{\beta}=-2 t_{+} \frac{R}{F} \Delta T \ln c^{\mathrm{T}}
\end{aligned}
$$




\section{S2. Preparation of SIM/PET Hybrid Nanochannels}

Preparation of SIM. SIM was firstly grown on the ITO glass using the Stöbersolution growth approach. ${ }^{\mathrm{S}}$ Briefly, the bare ITO glasses were immersed in the precursor solution containing $70 \mathrm{~mL}$ water, $30 \mathrm{~mL}$ ethanol, o.16 g CTAB, $10 \mu \mathrm{L}$ concentrated ammonia aqueous solution and $80 \mu \mathrm{L}$ TEOS. After $24 \mathrm{~h}$ under the quiescent condition at $60{ }^{\circ} \mathrm{C}$, SIM with $\mathrm{CTAB}$ micelles confined in silica nanochannels was formed on the ITO surface (designated as CTAB@SIM/ITO), and then aged at $100{ }^{\circ} \mathrm{C}$ overnight. The $\mathrm{CTAB}$ micelles in the silica nanochannels were removed by immersing the CTAB@SNM/ITO in o.1 $\mathrm{M} \mathrm{HCl}$ ethanol solution for $15 \mathrm{~min}$ under stirring to obtain micelle-free electrode (designated as $\mathrm{SIM} / \mathrm{ITO})$.

Preparation of PET with Conical Nanochannels. Poly(ethylene terephalate) membrane (PET, thickness: $12 \mu \mathrm{m}$, pore density: $10^{8}$ pores $\mathrm{cm}^{-2}$ ), irradiated with heavy ions, was treated by UV light (at $254 \mathrm{~nm}$ ) for $1 \mathrm{~h}$ before etching. The PET membrane was subsequently chemically etched from one side by $9 \mathrm{M} \mathrm{NaOH}$ solution, whereas the other side was put in contact with stopping solution containing $1 \mathrm{M} \mathrm{KCl}$ and $1 \mathrm{M} \mathrm{HCOOH}$ as neutralization agents. The etching process was performed at about $303 \mathrm{~K}$ and the trans-membrane ionic current at voltage of $1 \mathrm{~V}$ was monitored. The etching was stopped when an obvious current increase was observed. The geometry of prepared PET was characterized by SEM and current-voltage (Figure $\mathbf{2 c}, \mathbf{d}$ in the manuscript).

Preparation of Hybrid SIM/PET Nanochannels. SIM/PET hybrid nanochannels membrane was prepared using the PMMA-assisted transfer approach as previously reported, ${ }^{\mathrm{S}_{2}}$ as illustrated in Figure S1. Briefly, PMMA solution (3.5\% wt in anisole) was spin-coated on the top surface of SIM/ITO at $2000 \mathrm{rpm}$ for $30 \mathrm{~s}$. After solvent evaporation at room temperature for $1 \mathrm{~h}$ and heating at $115{ }^{\circ} \mathrm{C}$ for $15 \mathrm{~min}$, the obtained PMMA protected SIM/ITO was treated with $2 \mathrm{M} \mathrm{HCl}$ to etch the ITO layer and the free-standing PMMA/SIM was 
obtained. Subsequently, a piece of PET membrane with conical nanochannels was used as the substrate to fish out the free-standing PMMA/SIM. Finally, the top PMMA layer was dissolved in the mixture of toluene and acetone $(\mathrm{V} / \mathrm{V}=10 / 1)$ for $12 \mathrm{~h}$ to obtain the hybrid nanochannel membrane with perforated channels.
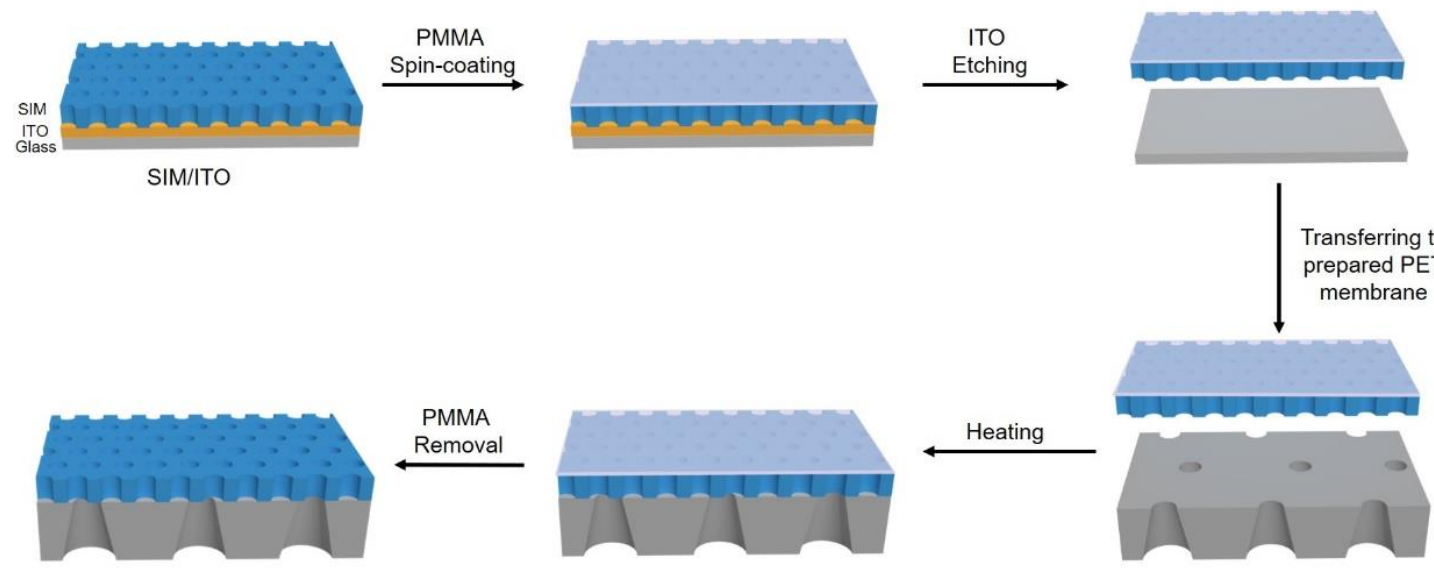

SIM/PET

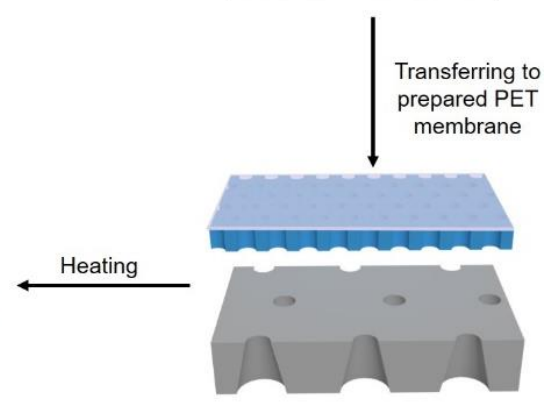

Prepared PET substrate

Figure S1. Schematic illustration of the preparation of SIM/PET hybrid nanochannels. 


\section{S3. I-V Response of Nanochannels}

For investigating ion transport property of nanochannels, the ion current was recorded by a Keithley 6847 picoammeter (Keithley Instruments, Cleveland, $\mathrm{OH}$ ) with a custom-made electrochemical cell. A pair of $\mathrm{Ag} / \mathrm{AgCl}$ electrodes is used to supply voltage and to measure the current. The voltage was scanned from $-1.0 \mathrm{~V}$ to $1.0 \mathrm{~V}$ with a step of $0.1 \mathrm{~V}$ and a period of $30 \mathrm{~s}$.

Figure S2 compares the current-voltage $(I-V)$ characteristic of PET conical nanochannels and SIM/PET hybrid nanochannels in $10 \mathrm{mM} \mathrm{KCl}$. Apparently, an enhanced ionic current rectification was observed, with the rectification ratio $\left(\left|I_{+1.0} \mathrm{~V} / I_{-1.0} \mathrm{~V}\right|\right)$ increased from 3.6 to 9.1, confirming the successful preparation of layered nanochannel structure. The high pore density, ultra-small thickness and high surface charge density (provided by $\mathrm{Si}-\mathrm{OH}$ hydrolysis) of silica nanochannels are responsible of a better ion-selectivity of hybrid structure. Moreover, the ionic current did not decrease drastically (from $24.5 \mathrm{nA}$ to $16.5 \mathrm{nA}$ at +1.0 V) after SIM was adhered to PET nanochannels, indicating that silica nanochannels have a negligible effect on the ion permeability thanks to its high pore density.

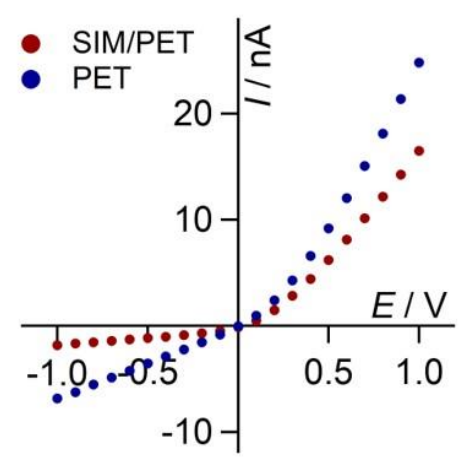

Figure S2. Comparison of $I-V$ responses of PET conical nanochannels (blue) and SIM/PET hybrid nanochannels (red). 


\section{S4. Thermoelectric Response of PET Conical Nanochannels}

The thermoelectric response of PET conical nanochannels was also measured to compare with that of SIM/PET hybrid nanochannels. Figure S3a shows the synchronous time evolution of $\Delta T$ and $\Delta V_{\text {oc }}$ recorded with PET conical nanochannels. The variation of $\Delta V_{\text {oc }}$ with $\Delta T$ is plotted in Figure $\mathbf{S}_{\mathbf{3}} \mathbf{b}$, which is also linearly fitted to eq. 5, yielding the sensitivity of thermosensation of 0.44 $\mathrm{mV} / \mathrm{K}$. From the slope, $t_{+}$(for $\mathrm{K}^{+}$in this case) can be calculated to be 0.55 . Both sensitivity and $t_{+}$value are smaller than those obtained with SIM/PET hybrid nanochannels, indicating a less permselective nature of PET conical nanochannels.
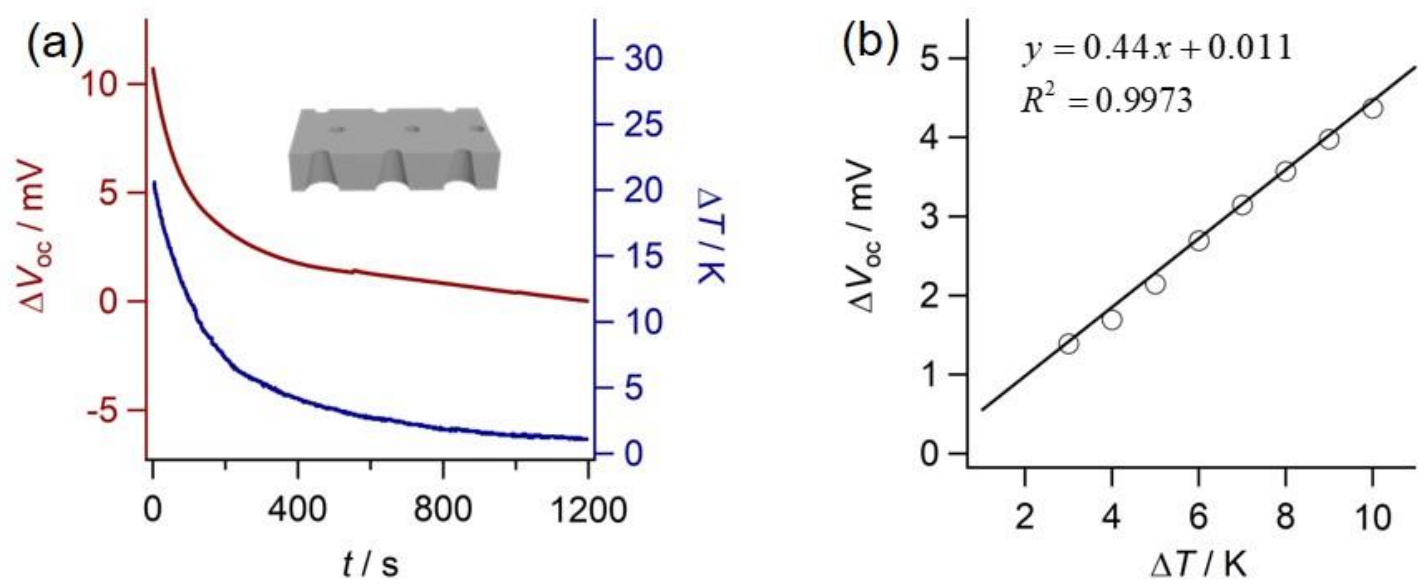

Figure $S_{3}$. Thermoelectric response of PET conical nanochannels. (a) The synchronous time evolution curves of $\Delta T$ (blue curve) and $\Delta V_{\text {oc }}$ (red curve) in $10 \mathrm{mM} \mathrm{KCl}$. The solution put into contact with the tip face was heated. (b) $\Delta \mathrm{V}_{\text {oc }}$ as function of $\Delta T$. The solid line represents the linear fitting by eq. 5 . 


\section{S5. Thermoelectric Response at a Low pH}

The thermoelectric response of SIM/PET hybrid nanochannels at a low $\mathrm{pH}$ (3.0) was measured to verify that the sensitivity is mainly contributed by the cationic selectivity of nanochannel. Figure $\mathbf{S} 4 \mathbf{a}$ shows the synchronous time evolution of $\Delta T$ and $\Delta V_{\text {oc }}$ recorded with SIM/PET hybrid nanochannels in $10 \mathrm{mM} \mathrm{KCl}(\mathrm{pH}=$ 3.o was adjusted by $\mathrm{HCl}$ ). The variation of $\Delta V_{\mathrm{oc}}$ with $\Delta T$ is plotted in Figure $\mathbf{S}_{\mathbf{4}} \mathbf{b}$, yielding the sensitivity of thermosensation of $0.52 \mathrm{mV} / \mathrm{K}$. From the slope, $t_{+}$(for $\mathrm{K}^{+}$in this case) was calculated to be 0.66 . These two values are apparently smaller than those obtained in the manuscript (10 $\mathrm{mM} \mathrm{KCl}, \mathrm{pH}=5.9)$. The decrease of both sensitivity and t can be ascribed to the decrease of surface charge density, due to protonation of carboxylic groups on PET surface and less deprotonation of silanol groups on silica nanochannel surface. The experimental also proved that the cationic selectivity is a decisive factor to achieve a high thermosensation sensitivity.

(a)

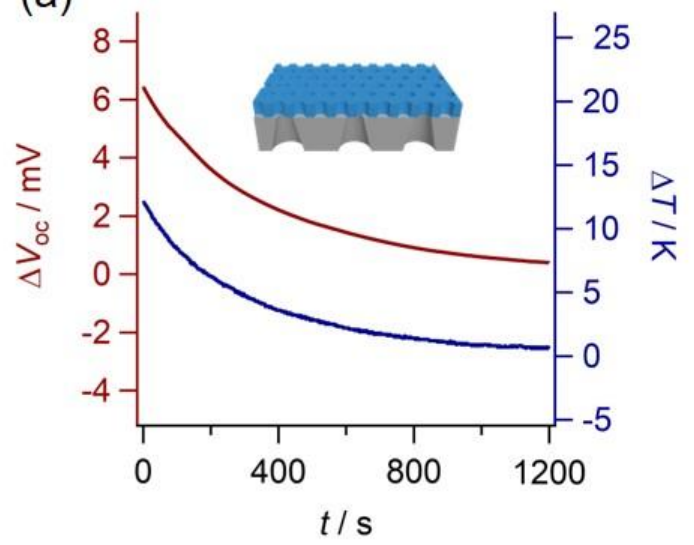

(b)

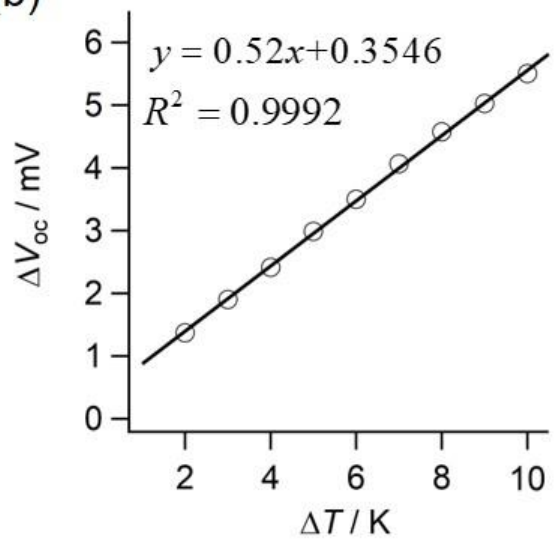

Figure S4. Thermoelectric response of SIM/PET hybrid nanochannels at $\mathrm{pH}=$ 3.o. (a) The synchronous time evolution curves of $\Delta T$ (blue curve) and $\Delta V_{\text {oc }}$ (red curve) in 10 $\mathrm{mM} \mathrm{KCl}(\mathrm{pH}=3.0)$. (b) $\Delta \mathrm{V}_{\mathrm{oc}}$ as function of $\Delta T$. The solid line represents the linear fitting by eq. 5 . 


\section{S6. Redox Potential Difference of Two AgCl/Ag Electrodes}

As shown in Figure $\mathbf{1 b}$, the thermoelectric response is measured with two $\mathrm{AgCl} / \mathrm{Ag}$ electrodes. According to the theoretical derivation in the first section, the measured value of $\Delta V_{\mathrm{oc}}$ includes the contributions from the variations of both $\phi_{\text {diff }}$ and $E_{\text {redox }}$. In order to determine the former, we need measure experimentally the latter. In terms of eq. $\mathbf{S 8}$, at the initial state $\left(T=T_{\mathrm{o}}\right), E_{\text {redox }}$ is solely determined by the concentration gradient. Upon changing the temperature $\left(\Delta T=T^{\beta}-T^{\alpha}\right)$, $E_{\text {redox }}$ will change and the magnitude of variation is given by,

$$
\begin{aligned}
\Delta E_{\text {redox }}(c, T) & =E_{\text {redox }}(c, T)-E_{\text {redox }}(0) \\
& =\frac{R T^{\beta}}{F} \ln c^{\beta}-\frac{R T^{\alpha}}{F} \ln c^{\alpha}-\frac{R T^{0}}{F} \ln c^{\beta}+\frac{R T^{0}}{F} \ln c^{\alpha} \\
& =\frac{R}{F} \Delta T \ln c^{\mathrm{T}}
\end{aligned}
$$

To measure $\Delta E_{\text {redox }}$, a home-made saturated salt bridge was used. As shown in Figure $\mathbf{S}_{\mathbf{5}}$, the experimental curve of $\Delta E_{\text {redox }}$ as a function of temperature variation, $\Delta T$, coincides with the theoretical one given by eq. $\mathrm{S} 18$. It proves that we can estimate $\Delta \phi_{\text {diff }}$ by subtracting $\Delta E_{\text {redox }}$ from $\Delta V_{\text {oc }}$.

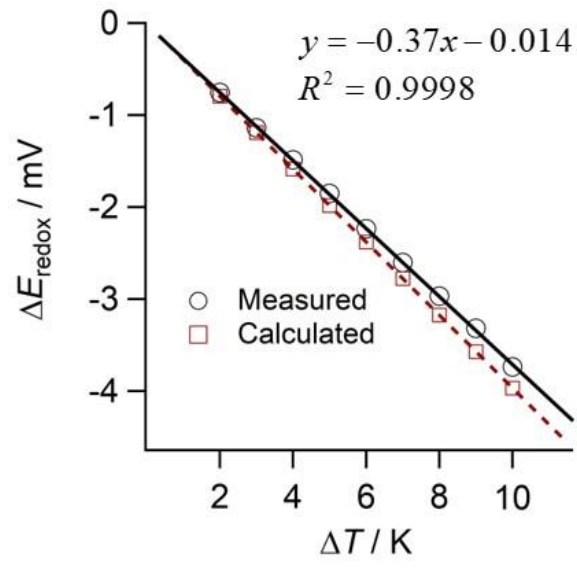

Figure $S_{5}$. The difference of redox potentials between two $\mathrm{Ag} / \mathrm{AgCl}$ electrodes $\left(\Delta E_{\text {redox }}\right)$. as a function of temperature variation $(\Delta T)$. 


\section{S7. Numerical Simulations}

Simulations of ion transport in the SIM/PET hybrid nanochannels were carried out by the commercial finite-element software COMSOL Multiphysics 5.2. For simplicity, a 2D axis symmetric model was employed in this system, as shown in

\section{Figure S6.}

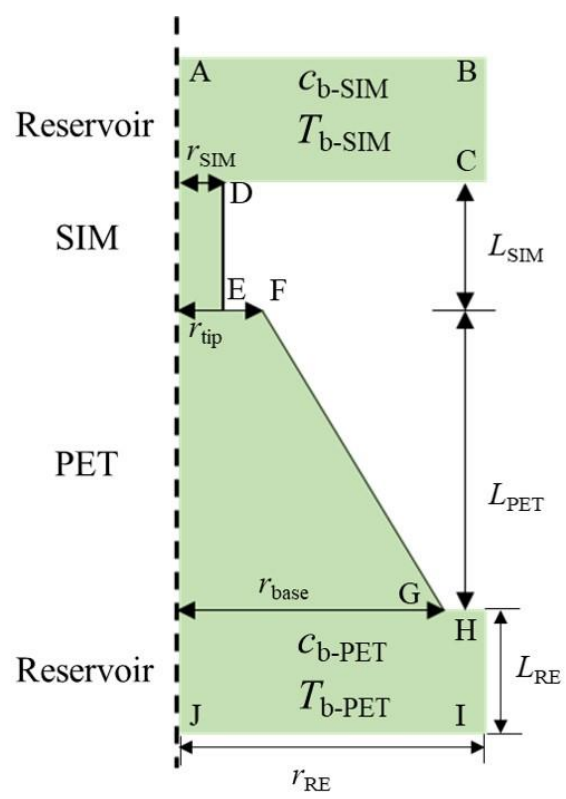

Figure S6. Scheme of 2D axial symmetric model with labeled boundaries (not to scale).

Here a conically-shaped PET channel with a tip opening radius of $r_{\text {tip }}=7 \mathrm{~nm}$, a base opening radius of $r_{\text {base }}=400 \mathrm{~nm}$, and a channel length of $L_{\mathrm{PET}}=12000 \mathrm{~nm}$ is considered. The SIM is positioned on the side of tip, which consists of nanochannels with a radius of $r_{\mathrm{SIM}}=1.15 \mathrm{~nm}$ and a length of $L_{\mathrm{SIM}}=100 \mathrm{~nm}$. The surface charge density of PET channel ( $\left.\sigma_{\mathrm{PET}}\right)$ and SIM nanochannel ( $\left.\sigma_{\mathrm{SIM}}\right)$ are set to $-0.002 \mathrm{C} / \mathrm{m}^{2}$ and $-0.015 \mathrm{C} / \mathrm{m}^{2}$, respectively. This SIM/PET hybrid nanochannel is connected to two large identical reservoirs with dimensions of 100 times of $r_{\text {base, }}$ 
which are filled with aqueous $\mathrm{KCl}$ or $\mathrm{NaCl}$ solutions. The boundary conditions in numerical simulation are summarized in Table S1.

Table S1. Boundary conditions for 2D axis symmetric model.

\begin{tabular}{|c|c|c|}
\hline Boundary & Poisson equation & Nernst-Planck equation \\
\hline $\mathrm{AB}$ & applied voltage, $\phi=V$ & $\begin{array}{l}\text { bulk concentration } \\
c_{+}=c_{-}=c_{\mathrm{b}-\text { SIM }}\end{array}$ \\
\hline JI & grounded, $\phi=0$ & $\begin{array}{l}\text { bulk concentration } \\
c_{+}=c_{-}=c_{\text {b-PET }}\end{array}$ \\
\hline $\mathrm{BC}$ & $-\mathbf{n} \cdot \nabla \phi=\mathbf{o}$ & $\begin{array}{l}\text { bulk concentration } \\
c_{+}=c_{-}=c_{\text {b-SIM }}\end{array}$ \\
\hline $\mathrm{HI}$ & $-\mathbf{n} \cdot \nabla \phi=\mathbf{o}$ & $\begin{array}{l}\text { bulk concentration } \\
c_{+}=c_{-}=c_{\text {b-PET }}\end{array}$ \\
\hline $\mathrm{CD}, \mathrm{GH}$ & $-\mathbf{n} \cdot \nabla \phi=\mathbf{o}$ & $\begin{array}{l}\text { ion-impenetrable } \\
\mathbf{n} \cdot \boldsymbol{j}_{i}=\mathbf{0}\end{array}$ \\
\hline DE, EF & $-\boldsymbol{c} \mathbf{n} \cdot \nabla \phi=\sigma_{\mathrm{SIM}}$ & $\begin{array}{l}\text { ion-impenetrable } \\
\mathbf{n} \cdot \boldsymbol{j}_{i}=\mathbf{0}\end{array}$ \\
\hline GH & $-\boldsymbol{\varepsilon n} \cdot \nabla \phi=\sigma_{\mathrm{PET}}$ & $\begin{array}{l}\text { ion-impenetrable } \\
\mathbf{n} \cdot \boldsymbol{j}_{i}=\mathbf{o}\end{array}$ \\
\hline
\end{tabular}

n: the unit outer normal vector.

The coupled Poisson-Nernst-Planck (PNP) and Einstein-Stokes equations are employed to quantitively describe the ionic mass transport process,

$$
\begin{aligned}
& \nabla^{2} \phi=-\frac{\rho}{\varepsilon}=-\frac{F}{\varepsilon} \sum z_{i} c_{i} \\
& \mathbf{j}_{i}=-D_{i}\left(\nabla c_{i}+\frac{z_{i} F c_{i}}{R T} \nabla \phi\right) \\
& \nabla \cdot \mathbf{j}_{i}=0 \\
& D_{i}=\frac{k_{\mathrm{B}} T}{6 \pi \eta r}
\end{aligned}
$$

where 
$\phi[\mathrm{V}]:$ electric potential;

$\rho\left[\mathrm{C} / \mathrm{m}^{3}\right]$ : space charge density;

$\mathbf{j}_{i}\left[\mathrm{~mol} /\left(\mathrm{m}^{2} \mathrm{~s}\right)\right]:$ ionic flux;

$z_{i}[-]$ : charge number;

$\mathrm{C}_{i}[\mathrm{M}]$ : ion concentration;

$D_{i}\left[\mathrm{~m}^{2} / \mathrm{s}\right]$ : diffusion coefficient;

$\varepsilon=78.5[\mathrm{~F} / \mathrm{m}]$ : dielectric constant of water;

$F[\mathrm{C} / \mathrm{mol}]$ : Faraday constant;

$R[\mathrm{~J} / \mathrm{K} / \mathrm{mol}]$ : gas constant;

$k_{\mathrm{B}}$ : Boltzmann constant;

$T[\mathrm{~K}]$ : absolute temperature;

$\eta\left[\mathrm{Pa}^{*} \mathrm{~s}\right]$ : the dynamic fluid viscosity of water, expressed by a built-in piecewise function in COMSOL;

$r$ is the ion size $\left(\right.$ for $\mathrm{K}^{+} / \mathrm{Cl}^{-}, r=0.3 \mathrm{~nm}$; for $\left.\mathrm{Na}^{+}, r=0.4 \mathrm{~nm}\right) ; \mathrm{S}_{3}$

$i=+\left(\right.$ for $\mathrm{K}^{+}$or $\left.\mathrm{Na}^{+}\right)$

$i=-\left(\right.$ for $\left.\mathrm{Cl}^{-}\right)$.

The models in the absence and in the presence of a concentration gradient were calculated to explore the origin of temperature-responsive electric signal. We plot the ionic concentration profile $\left(c_{\mathrm{z}}\right.$, for both $\mathrm{K}^{+}$and $\left.\mathrm{Cl}^{-}\right)$and electric field $\left(E_{\mathrm{z}}\right)$ along the axial direction of nanochannel before and after a temperature change of $10 \mathrm{~K}$. The initial temperature of two solutions is set at $283.15 \mathrm{~K}\left(T_{\mathrm{b}-\mathrm{SIM}}=T_{\mathrm{b} \text {-PET }}\right.$ $=283.15 \mathrm{~K})$.

In the absence of a concentration gradient, $T_{\mathrm{b} \text {-SIM }}$ is increased by $10 \mathrm{~K}$ to $293.15 \mathrm{~K}$, while $T_{\text {b-PET }}$ remains unchanged at $283.15 \mathrm{~K}$. For simplicity, a sharp temperature boundary is assumed to exist at the SIM/reservoir solution interface. As shown in Figure $S_{7} \mathbf{a}$, b, due to the highly cationic permselectivity of SIM nanochannel, there exists a remarkable accumulation of $\mathrm{K}^{+}$and an obvious depletion of $\mathrm{Cl}^{-}$ 
inside. Although PET channel is larger, we still observe a slightly depletion of $\mathrm{Cl}^{-}$ inside. Moreover, it is clear that there exists two distinct asymmetric profiles of $\mathrm{K}^{+}$and $\mathrm{Cl}^{-}$at the orifice of SIM nanochannel and at the boundary of PET/SIM, which results in negative and positive overshoots of $E_{\mathrm{z}}$, respectively, as shown in Figure $S_{7} c$. However, the variations of both $c_{z}$ and $E_{z}$ in response to the temperature change are too small to recognize directly in Figure $\boldsymbol{S}_{\mathbf{7}}$. So we analyze their net variations, namely $\Delta c_{\mathrm{z}}$ and $\Delta E_{\mathrm{z}}$, as shown in Figure $\mathbf{4} \mathbf{b}, \mathbf{c}$.
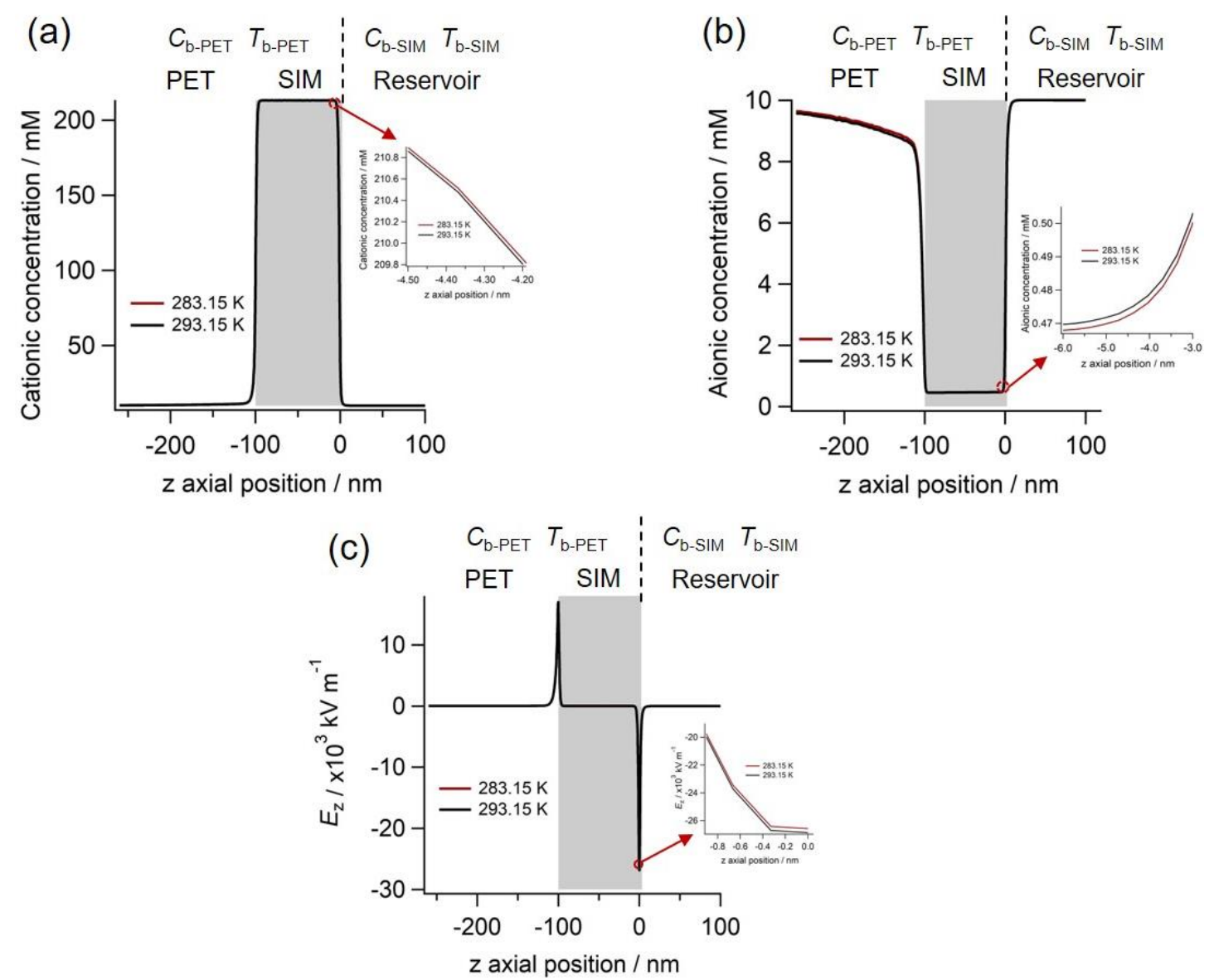

Figure $\mathrm{S}_{7}$. (a, b) Calculated ion concentration profiles of $\mathrm{K}^{+}(\mathrm{a})$ and $\mathrm{Cl}^{-}(\mathrm{b})$ along the axial direction of hybrid nanochannel before (red curve) and after (black curve) the temperature change. (c) Calculated $E_{\mathrm{z}}$ along the axial direction of hybrid nanochannel for two cases. The gray region $(-100 \mathrm{~nm} \leq \mathrm{z} \leq 0 \mathrm{~nm})$ refers to SIM, the region $(-250 \mathrm{~nm}$ $\leq \mathrm{z} \leq-100 \mathrm{~nm})$ denotes PET, and the region (o $\mathrm{nm} \leq \mathrm{z} \leq 100 \mathrm{~nm}$ ) represents the reservoir solution, respectively. The electrolyte is $10 \mathrm{mM} \mathrm{KCl}$. $T_{\mathrm{b}-\text {-siM }}$ is increased by $10 \mathrm{~K}$ from $283.15 \mathrm{~K}$ to $293.15 \mathrm{~K}$, whereas $T_{\mathrm{b} \text {-PET }}$ remains unchanged at $283.15 \mathrm{~K}$. The insets are the magnifications at the orifice of nanochannel. 

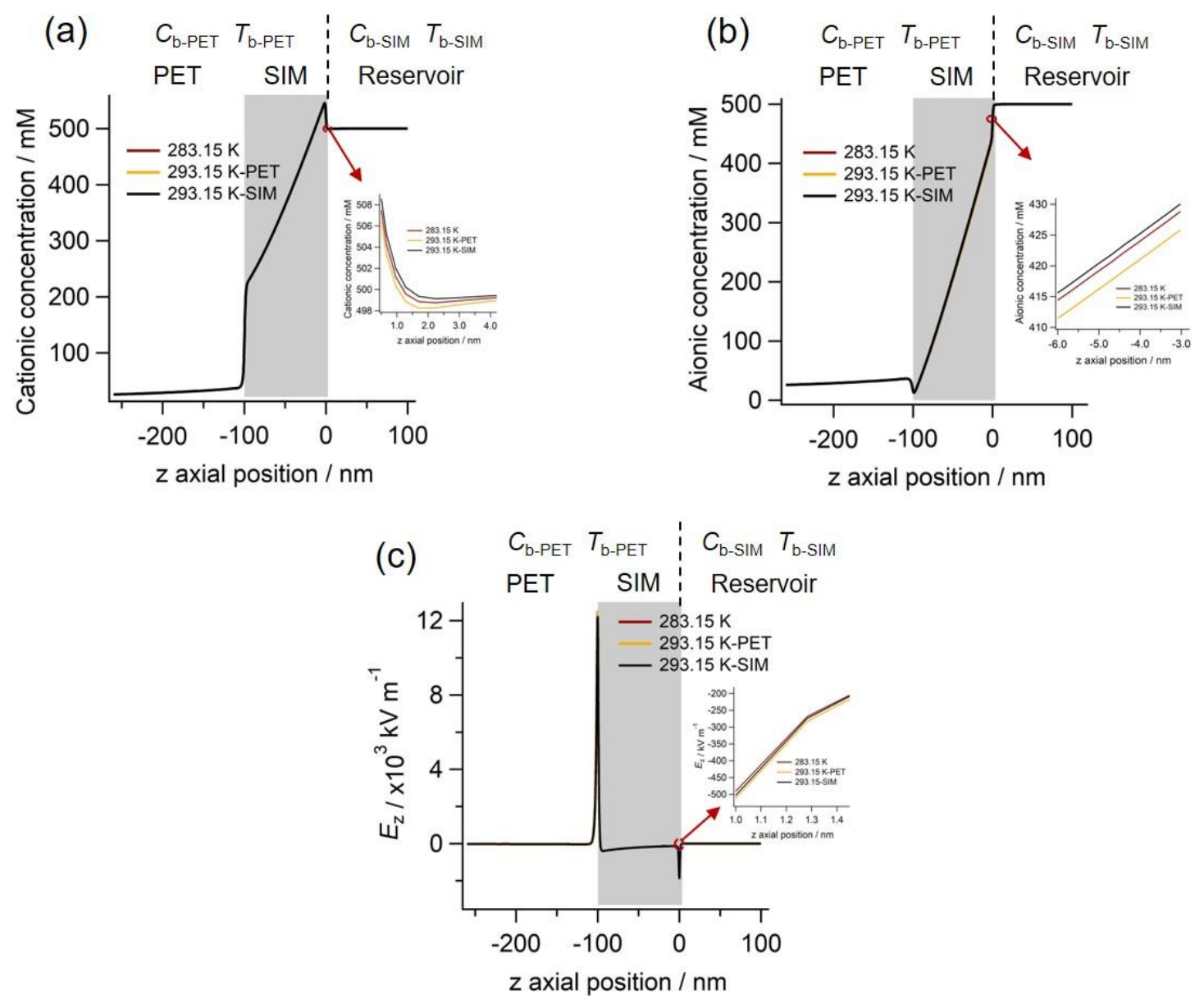

Figure S8. (a, b) Calculated ion concentration profiles of $\mathrm{Na}^{+}(\mathrm{a})$ and $\mathrm{Cl}^{-}(\mathrm{b})$ along the axial direction of hybrid nanochannel before temperature change (red curve) and after the temperature change in the solution contacted with PET (low electrolyte concentration, yellow curve) and in the one with SIM nanochannel (high electrolyte concentration, black curve). (c) Calculated $E_{\mathrm{z}}$ along the axial direction of hybrid nanochannel for three cases. The gray region $(-100 \mathrm{~nm} \leq \mathrm{z} \leq \mathrm{o} \mathrm{nm})$ refers to SIM, the region $(-250 \mathrm{~nm} \leq \mathrm{z} \leq-100 \mathrm{~nm}$ ) denotes PET, and the region (o $\mathrm{nm} \leq \mathrm{z} \leq 100 \mathrm{~nm}$ ) represents the reservoir solution, respectively. $c_{\mathrm{b} \text {-PET }}$ and $c_{\mathrm{b} \text {-SIM }}$ are set at $10 \mathrm{mM}$ and 500 $\mathrm{mM}$, respectively. $T_{\mathrm{b} \text {-SIM }}$ and $T_{\mathrm{b} \text {-PET }}$ are set at $283.15 \mathrm{~K}$, then one of which is increased by $10 \mathrm{~K}$ to $293.15 \mathrm{~K}$ and the other remains unchanged at $283.15 \mathrm{~K}$. The insets are the magnifications at the orifice of nanochannel.

In the presence of a concentration gradient, either $T_{\mathrm{b} \text {-SIM }}$ or $T_{\mathrm{b}-\mathrm{PET}}$ is increased by 10 $\mathrm{K}$ to $293.15 \mathrm{~K}$, whereas the other one remains unchanged at $283.15 \mathrm{~K}$. For simplicity, it is assumed that the sharp temperature boundary is located at the orifice of nanochannels in both cases $(z=0)$. Figure S8a, $\mathbf{b}$ shows the concentration profiles of ions (for both $\mathrm{Na}^{+}$and $\mathrm{Cl}^{-}$) before and after the temperature changes. Because the SIM nanochannel is put in contact with the 
concentrated solution (o.5 M NaCl), mild concentration gradients for both $\mathrm{Na}^{+}$ and $\mathrm{Cl}^{-}$are observed at the orifice of nanochannel, arising from the weaker EDL effect. The direction of concentration gradients for $\mathrm{Na}^{+}$and $\mathrm{Cl}^{-}$is opposite, leading to a slight negative overshoot of $E_{\mathrm{z}}$ at this position (see Figure S8c). Inside the SIM nanochannel, although $\mathrm{Na}^{+}$and $\mathrm{Cl}^{-}$both deplete toward the low concentration solution side, the depletion of $\mathrm{Cl}^{-}$is more significant, which is because the cationic selectivity of nanochannel turns better and better gradually toward this direction. So a faster depletion of $\mathrm{Na}^{+}$appears at the boundary of PET/SIM, leading to a sharp positive overshoot of $E_{\mathrm{z}}$ in the electric field profile. Note that there exists a slight accumulation of $\mathrm{Cl}^{-}$at the orifice of PET nanochannel nearby the boundary, due to the better cationic selectivity of SIM nanochannel than PET channel.

However, the variations of both $c_{\mathrm{z}}$ and $E_{\mathrm{z}}$ in response to the temperature change in two cases are too small to recognize directly in Figure S8. So we also analyze their net variations, namely $\Delta c_{\mathrm{z}}$ and $\Delta E_{\mathrm{z}}$, as shown in Figure 6a, b, $\mathbf{c}$. 


\section{References}

(S1) Teng, Z.; Zheng, G.; Dou, Y.; Li, W.; Mou, C.-Y.; Zhang, X.; Asiri, A. M.; Zhao, D. Highly Ordered Mesoporous Silica Films with Perpendicular Mesochannels by a Simple Stöber-Solution Growth Approach. Angew. Chem., Int. Ed. 2012, 51, 2173.

(S2)Wu, W.; Yang, Q.; Su, B. Centimeter-scale continuous silica isoporous membranes for molecular sieving. J. Membr. Sci. 2018, 558, 86.

(S3) Duan, C.; Majumdar, A. Anomalous ion transport in 2-nm hydrophilic nanochannels. Nat. Nanotechnol. 2010, 5, 848. 